02

\title{
Сильная интерференционная люминесценция смешанных мод в окрестности критического значения затухания экситона
}

\author{
(C) Б.Ж. Ахмадалиев, Н.Х. Юлдашев \\ Ферганский политехнический институт, \\ 150107 Фергана, Узбекистан \\ ฯ e-mail: uzferfizika@mail.ru
}

Поступила в редакцию 27.10.2020 г.

В окончательной редакции 23.04.2021 г.

Принята к публикации 18.05.2021 г.

\begin{abstract}
Проанализированы результаты численного расчета дисперсии и спектров люминесценции смешанных экситонных мод кристаллов типа $\mathrm{CdS}\left(A_{n-1}\right)$ в зависимости от затухания Г механических экситонов и угла выхода $\theta$ излучения в вакуум в окрестности критических значений $\Gamma_{c}$ и $\theta_{c}$. Показано, что механизм формирования линии $A_{L}$ существенно зависит от значения параметра свето-экситонного взаимодействия $\tilde{\omega}_{L T}\left(\theta_{c}\right) / \Gamma_{c}$, суперпозиция волн 1 и 2 с сильно асимметричными $I_{M 1}(\omega), I_{M 2}(\omega)$ и симметричным $I_{12}(\omega)$ контурами формирует практически симметричный результирующий спектральный контур $I(\omega)$ с максимумом на критической частоте $\omega_{c}$.
\end{abstract}

Ключевые слова: экситон-поляритоны, смешанные моды, пространственная дисперсия, аномальная дисперсия, промежуточное свето-экситонное взаимодействие, затухание экситона, интерференционная люминесценция.

DOI: $10.21883 / \mathrm{OS} .2021 .09 .51339 .268-20$

\section{Введение}

Настоящая работа посвящена исследованию особенностей формирования дисперсии и спектров фотолюминесценции смешанных экситон-поляритонных мод (СЭПМ) гексагональных кристаллов типа $\mathrm{CdS}\left(A_{n-1}\right)$ в окрестности критического значения затухания $\Gamma \approx \Gamma_{c}$ механического экситона. Хотя экситонные дисперсии, поглощение и люминесценция подробно рассмотрены во многих работах (см., например, [1-5]), тем не менее отсутствует необходимая информация о свойствах смешанного экситона в условиях, когда $\Gamma \rightarrow \Gamma_{c}$. В кристалле $\mathrm{CdS}$ смешанные моды обнаруживаются при анизотропной геометрии излучения экситонов, волновой вектор которых составляет некоторый угол $\theta$ (отличный от $0^{\circ}$ и $90^{\circ}$ ) с напряженностью электрического поля и гексагональной осью $C_{6}$. Изменяя угол от $0^{\circ}$ до $90^{\circ}$, можно плавно изменять значение ,эффективного продольно-поперечного расщепления“ $\tilde{\omega}_{L T}$ в одном и том же кристалле [2,4] и тем самым исследовать состояния системы „экситон + фотоны“ в кристалле с промежуточным свето-экситонным взаимодействием (СЭВ) $\omega_{L T} \sim \Gamma$.

Как известно [3,6,7], для поперечных поляритонов с изотропной эффективной массой $M$ экситона и резонансной частотой $\omega_{0}$, когда диссипативное затухание экситона $\Gamma=\Gamma_{c}=4\left(\varepsilon_{b} \omega_{L T} \omega_{M}\right)^{1 / 2} \quad\left(\right.$ где $\varepsilon_{b}-$ фоновая диэлектрическая проницаемость кристалла, $\left.\omega_{M}=\hbar k_{0}^{2} / 2 M\right)$, дисперсионное уравнение на частоте $\omega=\omega_{c}=\omega_{0}+\varepsilon_{b} \omega_{M}$ имеет кратные корни относительно $k^{2}$. Это также имеет место и для дисперсии смешанных мод $[2,4,5]$, что приводит к существенному перемешиванию квантовых состояний и к сильному интерференционному излучению когерентных состояний нижней $M 1$ и верхней $M 2$ дисперсионных ветвей в окрестности частоты пересечения $\omega_{c}$ в зависимости от $\Gamma$ и угла выхода $\theta$ излучения в вакуум. Данный случай интересен, например, для создания источников когерентного излучения одиночных фотонов на основе новых принципов спектроскопии СЭПМ. Ниже приводим результаты анализа численного расчета дисперсии и спектров фотолюминесценции смешанных мод кристаллов типа $\mathrm{CdS}$ в зависимости от угла $\theta=\theta_{c}$.

\section{Теория}

В работах [4,8] показано, что в окрестности критического значения (для параметров кристалла $\mathrm{CdS}$ ) максимальное значение $\hbar \Gamma_{c} \approx 0.5 \mathrm{meV}(T \approx 45 \mathrm{~K})$, и легко могут быть реализованы случаи промежуточного СЭВ и аномальной дисперсии, вызывающие сильное интерференционное излучение когерентных состояний смешанных мод с узкой спектральной шириной $\Delta \leq 0.1 \mathrm{meV}$. Мы воспользуемся формальным сходством между дисперсионными уравнениями поперечных и смешанных мод, обобщим результаты работы $[1,6]$ для изотропного поглощения света экситонами с учетом пространственной дисперсии (ПД, spatial dispersion, SD) на случай анизотропного поглощения. Решения дисперсионного уравнения для смешанных мод с учетом затухания 
можно записать как

$$
\begin{aligned}
& n_{M \beta}^{2}(\omega \theta)=\frac{1}{2}\left\{N(\omega, \theta)+\varepsilon_{b}-(-1)^{\beta}\right. \\
& \left.\times\left[\left[N(\omega, \theta)-\varepsilon_{b}\right]^{2}+4\left(\omega_{L T / \omega_{M}}\right) \sin ^{2} \theta\right]^{1 / 2}\right\},
\end{aligned}
$$

где $\beta=1,2, N=(\omega, \theta)=\left(1-M_{\perp} / M_{\|}\right) \sin ^{2} \theta+\left(\omega-\omega_{L}+\right.$ $+i \Gamma / 2), M_{\perp}$ и $M_{\|}$- поперечная и продольная эффективные массы, $\omega_{L}$ - частота продольного экситона. Для одноосных кристаллов типа $\mathrm{CdS}$ выполняется неравенство $\left(1-M_{\perp} / M_{\|}\right) \sin ^{2} \theta \ll \varepsilon-[b]$, и из (1) на частоте $\omega=\omega_{c} \simeq \omega_{L}+\omega_{M_{\perp}} \varepsilon_{b}\left(\right.$ где $\left.\omega_{M_{\perp}}=\hbar k_{0}^{2} / 2 M_{\perp}\right)$ получаем

$$
n_{M \beta}^{2}\left(\omega_{c}, \theta\right)=\varepsilon_{0}+\frac{\Gamma}{4 \omega_{M_{\perp}}}\left[i-(-l)^{\beta} \sqrt{\frac{\Gamma_{c}^{2}(\theta)}{\Gamma^{2}}-l}\right],
$$

где

$$
\Gamma_{c}(\theta)=\simeq 4 \sin \theta \sqrt{\omega_{M_{\perp}} \omega_{L T}}=4 \sqrt{\omega_{M_{\perp}} \tilde{\omega}_{L T} \varepsilon_{b}} .
$$

Здесь частота $\omega$ имеет простой геометрический смысл: она соответствует точке пересечения дисперсионных кривых поперечных фотонов в поляризации $\mathbf{E} \| \mathbf{C}$ и чисто продольных экситонов. На частоте $\omega=\omega_{c}$ при критическом значении затухания $\Gamma=\Gamma_{c}\left(\theta=\theta_{c}\right)$ из (2) получаются кратные корни $n_{M 1}=n_{M 2}$. Величина $\Gamma_{c}$ (3) содержит в качестве параметра эффективную массу экситона $M_{\perp}$, и, естественно, отношение характеризует величину ПД по отношению к затуханию, а с другой стороны, $\tilde{\omega}_{L T}(\theta) / \Gamma=\omega_{L T} \sin ^{2} \theta / \varepsilon_{b} \Gamma$ является аналогичной мерой СЭВ в спектре смешанных мод. При $M_{\perp} \rightarrow \infty,\left(M_{\|}\right) \rightarrow \infty$ и $\Gamma \neq 0$ имеем $\Gamma_{c} / \Gamma \ll 1$, что означает отсутствие ПД, причем для кристаллов с ярко выраженной ПД в изотропной геометрии может осуществиться $\Gamma \gg \Gamma$. В случае смешанных мод $\Gamma_{c}$, согласно (3), зависит от $\theta$, и для типичных значений оптических параметров кристалла $\mathrm{CdS}$ из (3) получим максимальное значение $\Gamma_{c} \approx 0.48 \mathrm{meV}\left(\tilde{\omega}_{L T} \approx 0.213 \mathrm{meV}\right)$ при $\theta=\pi / 2$, в то время как для изотропной геометрии $\Gamma_{c} \approx 1.457 \mathrm{meV}\left(\omega_{L T} \approx 2.0 \mathrm{meV}\right)$ в окрестности температур $T \approx 4 \mathrm{~K}$ [8]. Поэтому для смешанных мод эффекты ПД и СЭВ проявляются слабее по сравнению с поперечными поляритонами. Однако, как показано ниже, в отличие от последних в спектрах низкотемпературной фотолюминесценции СЭПМ обнаруживаются эффекты сильной анизотропии, аномальной дисперсии и гигантского интерференционного излучения.

Здесь особо следует отметить, что интерференционное излучение смешанных мод является одной из разновидностей проявления эффектов ПД [7]. Для сред без ПД величина $\Gamma_{c}=0$, и в таких средах отсутствует интерференционный эффект. Заметное интерференционное излучение экситон-поляритонных состояний наблюдается во всех кристаллах с ПД, когда $\Gamma \approx \Gamma_{c}=4 \sqrt{\omega_{M} \omega_{L T} \varepsilon_{b}}$, т. е.

$$
\frac{\omega_{L T}}{\Gamma} \approx \frac{1}{4 \sqrt{\varepsilon_{b} \omega_{M} / \omega_{L T}}} .
$$

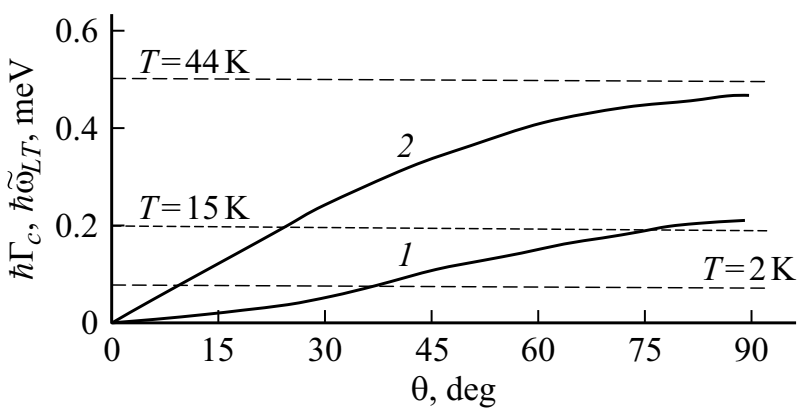

Рис. 1. Зависимости от угла выхода $\theta$ значений $\tilde{\omega}_{L T}(1)$ и $\Gamma_{c}(2)$, рассчитанные для параметров смешанных мод экситонного резонанса $A_{n-1}$ в CdS.

Для многих полупроводников с ярко выраженными экситонными резонансами знаменатель в формуле (4) $4 \sqrt{\varepsilon_{b} \omega_{M} / \omega_{L T}} \sim 1$, а, значит, рассматриваемый эффект практически обнаруживается в кристаллах с промежуточной величиной СЭВ.

Варьирование (за счет изменения $\theta$ ) параметров $\Gamma(\theta)$ и $\tilde{\omega}_{L T}(\theta)$ для одного и того же образца $\mathrm{CdS}$ при фиксированных значениях других параметров экситонного резонанса обеспечивает очень простой и надежный способ изучения эффектов, связанных с ПД и СЭВ. При этом появляется интересная с физической точки зрения возможность экспериментального сопоставления предельных и промежуточных ситуаций, в которых параметры Г и $\tilde{\omega}_{L T}$ могут принимать сильно отличающиеся от $\Gamma$ и сравнимые с $\Gamma$ значения. Сам параметр $Г$ может варьировать в определенных пределах, например, путем изменения температуры кристалла.

На рис. 1 построены рассчитанные зависимости от $\theta$ величин $\Gamma_{c}$ и $\tilde{\omega}_{L T}$ для типичных значений параметров экситонного резонанса $A_{n=1}$ в CdS. Этот рисунок позволяет сделать вывод, что в эксперименте действительно могут быть реализованы существенно разные соотношения между величинами $\Gamma, \Gamma_{c}$ и $\tilde{\omega}_{L T}$ : 1) $\left.\left.\Gamma \gg \Gamma_{c}, \Gamma \gg \tilde{\omega}_{L T}, 2\right) \Gamma \geq \Gamma_{c}, \Gamma \gg \tilde{\omega}_{L T}, 3\right) \Gamma \leq \Gamma_{c}$, $\left.\Gamma>\tilde{\omega}_{L T}, 4\right) \Gamma \ll \Gamma_{c}, \Gamma<\tilde{\omega}_{L T}$. Случай сильной экситонфотонной связи $\left(\Gamma \ll \tilde{\omega}_{L T}\right)$ сюда не включен из-за реальных ограничений на значения экситонного затухания в $\mathrm{CdS}, \Gamma>0.075 \mathrm{meV}$. Такой случай имеет место в геометрии поперечных мод $(\mathbf{E} \perp \mathbf{C}, \mathbf{k} \perp \mathbf{C})$, когда $\Gamma \ll \omega_{L T}$ (для $\mathrm{CdS} \hbar \tilde{\omega}_{L T} \leq 0.2 \mathrm{meV} \ll \hbar \omega_{L T} \approx 2 \mathrm{meV}$ при $T=2 \mathrm{~K})$. В предельном случае $\Gamma \ll \Gamma_{c}, \tilde{\omega}_{L T}$ эффекты ПД и СЭВ естественно не сказываются на формировании экситонного излучения, контур которого приобретает лоренцевский характер, а его полуширина определяется значением $\Gamma[4,8]$. В случае $\Gamma \sim \Gamma_{c}>\tilde{\omega}_{L T}$ наблюдается сильная интерференция состояний смешанных мод $\left(\mathbf{k}_{M 1} \approx \mathbf{k}_{M 2}\right)$ в условиях аномальной дисперсии, причем поляритонные эффекты несущественны. При $\Gamma<\Gamma_{c}$ и $\Gamma \leq \tilde{\omega}_{L T}$ эффекты ПД, СЭВ и интерференционное излучение когерентных состояний смешанных мод $M_{1}$ и 

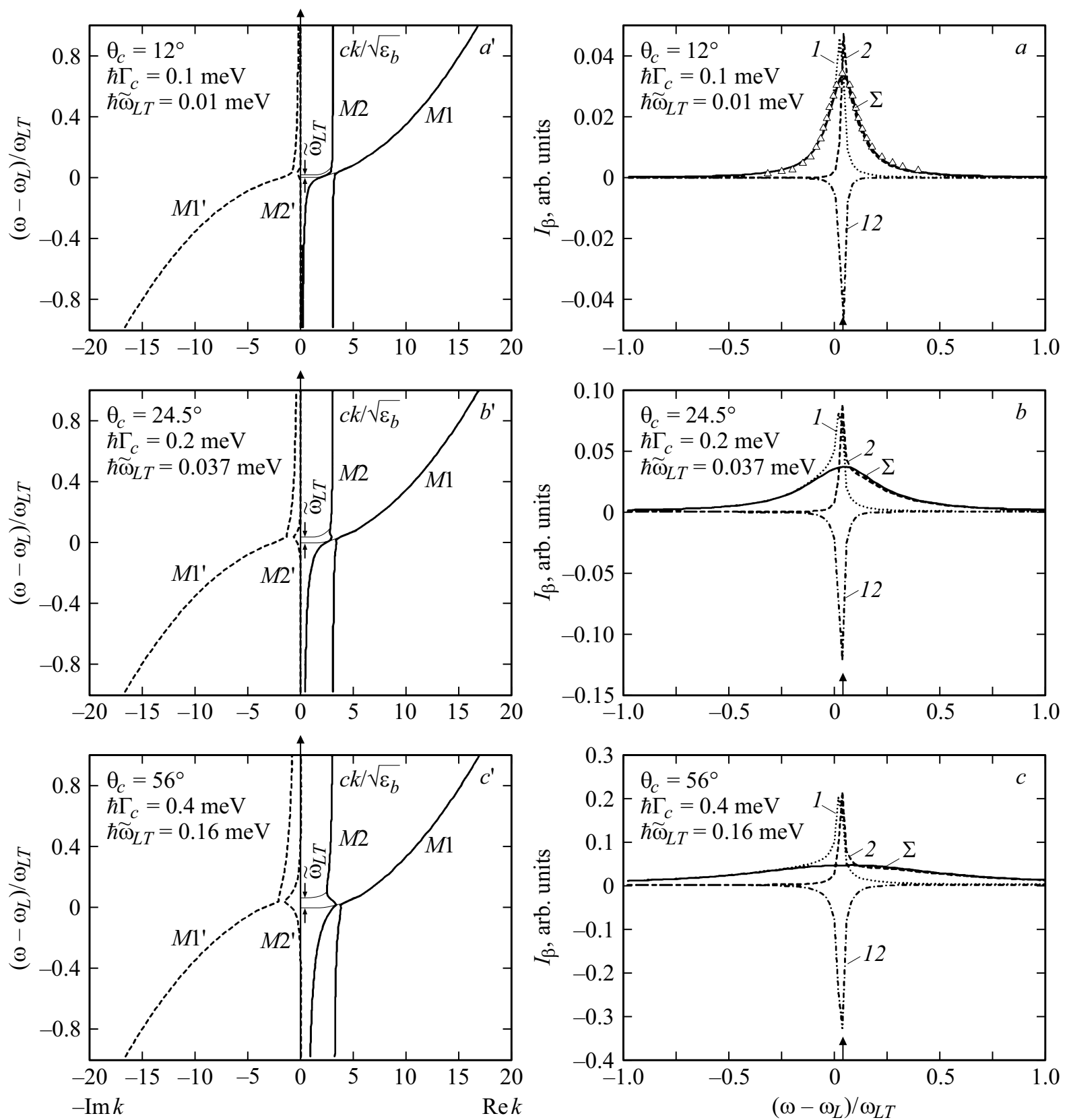

Рис. 2. Сравнение дисперсионных кривых $\left(a^{\prime}-c^{\prime}\right)$ и спектров фотолюминесценции $(a-c)$ смешанных мод при критических значениях $\Gamma_{c}=\Gamma$, рассчитанных для значений параметров $\mathrm{CdS}$ при $T=2 \mathrm{~K}$. Треугольники на рис. $a-$ эксперимент [4].

$M_{2}$ должны играть более умеренную роль в спектрах экситон-поляритонной люминесценции (ЭПЛ).

В работах $[3,4,6,7]$ была построена микроскопическая теория ЭПЛ с использованием диаграммной техники Келдыша в случае, когда диссипативное затухание экситона нарушает критерии применимости кинетического уравнения Больцмана для функции распределения экситон-поляритонов,

$$
\begin{gathered}
\left|\operatorname{Re} k_{\beta}\right| \gg \alpha \beta, \quad\left|\operatorname{Re}\left(k_{\beta}-k_{\beta^{\prime}}\right)\right| \gg \alpha \beta, \alpha \beta^{\prime}, \\
\left(\beta, \beta^{\prime}=1,2,3 \text { или } M 1, M 2\right) .
\end{gathered}
$$

Развитая теория, справедливая при произвольном соотношении между $\left|\operatorname{Re} k_{\beta}\right|$ и $\alpha \beta$ (где $k_{\beta}$ и $\alpha \beta-$ волновой вектор и коэффициент поглощения волны дисперсионной ветви $\beta$ ), позволила учесть интерференцию когерентно излучающих квантовых состояний поперечных поляритонов и продольных экситонов, а также смешанных мод при наличии затухания $(\Gamma \neq 0)$. На основе этой теории были последовательно рассчитаны вклады смешанных мод $M 1, M 2$ и их интерференции [4], а также нижней поляритонной ветви 1 , сильно затухающих волн 2 и 3 в ЭПЛ $[3,6,7]$ в окрестности частоты $\omega_{L}$ продольного экситона и удовлетворительно проанали- 
зированы экспериментальные спектры ЭПЛ кристаллов $\mathrm{CdS}, \mathrm{ZnP}_{2}$ и $\mathrm{CdTe}$.

Полученные в [4] формулы для парциальных интенсивностей $I_{\beta}(\omega, \theta)$ применимы при любых значениях $\Gamma_{c} / \Gamma$ и $\tilde{\omega}_{L T} / \Gamma$, когда $\Gamma \cong \Gamma_{\beta} \gg \tau_{M \beta}^{-1}$, где $\tau_{M \beta}-$ время внутризонного рассеяния для излучающих состояний смешанных мод. Однако в условиях сильной интерференции [8] таких состояний при $\Gamma \approx \Gamma_{c}\left(\theta \approx \theta_{c}\right)$ на частоте $\omega=\omega_{c}$ нельзя пользоваться этими формулами из-за наличия особенности $\left(n_{M 1}^{1}-N_{M 2}^{2}=0\right)$ в их знаменателях. Поэтому исключаем частоту $\omega_{c}$ при численном расчете $I_{\beta}(\omega \theta)$ и ограничиваемся значениями $\omega \rightarrow \omega_{c}$. Оказалось, что можно устранить эту особенность для суммарной интенсивности $I^{(0)}(\omega, \theta)=\sum_{\beta=1,2,12} I_{M \beta}(\omega \theta)$, и мы получили формулу

$$
I^{0}(\omega \theta)=C \frac{4 n_{o x}^{2} n_{0 z} L_{\mathrm{cr}}}{\left|N_{M}\right|^{2}} \Gamma_{f}(\omega),
$$

где введены следующие обозначения: $C=\frac{1}{(2 \pi)^{3}} \frac{2 M_{\perp}^{2} c \varepsilon_{b} \omega_{L T}}{\hbar}$, $c$ - скорость света в вакууме,

$$
\begin{gathered}
\bar{n}_{p}=\frac{N_{o z} \varepsilon_{b}\left(n_{M 1 z}+M_{M 2 z}\right)}{N_{0}^{2}\left(\varepsilon_{b}-N_{0}^{2}+n_{M 1 z} n_{M 2 z}\right)}, \\
\chi=\frac{n_{0 z} \varepsilon_{b}}{n_{0}^{2}\left(\varepsilon_{b}-n_{0 x}^{1 / 2}\right)} \delta=\varepsilon_{b} k_{0} l /\left(\varepsilon_{b}-n_{0 x}^{2}\right)^{1 / 2} n_{0}, \\
L_{c r}=L \frac{2 k_{0}^{2} L^{2}\left[l+k_{0} L\left(n_{M 1 z}^{\prime \prime}\right)+n_{M 2 z}^{\prime \prime}\right]}{\left(l+2 k_{0} L n_{M 1 z}^{\prime \prime}\right)\left(l+2 k_{0} L n_{m 2 z}^{\prime \prime}\right)}, \\
n_{0 x}=n_{0} \sin \theta, \quad n_{0 z}=n_{0} \cos \theta, \quad n_{M \beta z}^{\prime}=\operatorname{Re} n_{M \beta z}, \\
n_{M \beta z}^{\prime \prime}=\operatorname{Im} n_{M \beta z}, \quad n_{M \beta z}=\left(n_{M \beta}^{2}-n_{0 x}^{2}\right)^{1 / 2}, \\
\Gamma_{f}(\omega)=\sum_{\beta^{\prime}=T 1 M 1} \sum_{\mathbf{k}_{\beta^{\prime}}} \frac{f_{\beta^{\prime} \mathbf{k}_{\beta^{\prime}}}(\omega)}{\tau_{M \beta^{\prime} \mathbf{k}_{\beta^{\prime}}}(\omega)},
\end{gathered}
$$

$n_{0 x}$ и $n_{M \beta}=\left(k_{M \beta}^{2}\right)^{1 / 2} / k_{0}-$ показатели преломления окружающей среды и кристалла для мод $\beta=M^{1}, 2$, $k_{0}=\omega_{0} / C$ - длина мертвого (безэкситонного) слоя, $L$ - длина диффузии для мод $T 1$ и $M 1$ с достаточно большими волновыми векторами, состояния которых можно описать функциями распределения $f_{\beta^{\prime} \mathbf{k}_{\beta^{\prime}}}(\omega)$ и временами релаксации $\tau_{M \beta^{\prime} \mathbf{k}_{\beta^{\prime}}}^{\prime}(\omega)$ (см. формулу $(7)$ ). Поскольку мы интересуемся узким частотным интервалом $\Delta \hbar \omega \approx 0.25 \mathrm{meV}$, то при численном расчете спектров люминесценции СЭПМ пренебрегали плавной частотной зависимостью величин $f$ и Г.

\section{Анализ результатов численного расчета}

На рис. 2, $a^{\prime}, b^{\prime}, c^{\prime}$ представлены рассчитанные дисперсионные кривые СЭПМ в одноосном кристалле $\mathrm{CdS}$ при критических углах выхода излучения из кристалла в вакуум $\theta=12^{\circ}(a), 24.5^{\circ}(b), 56^{\circ}(c)$ и следующих значениях основных параметров экситона: $\hbar \Gamma=\hbar \Gamma_{c} \approx 0.1,0.2,0.4 \mathrm{meV}$ и $\hbar \omega_{0}=2552.4 \mathrm{meV}$, $\hbar \omega_{L T}=2 \mathrm{meV}, \quad \varepsilon_{b \perp}=9.4, \quad M_{\perp}=0.9 m_{0}, \quad M_{\|}=2.85 m_{0}$. Видно, что на частоте $\omega_{c}$ дисперсионные линии $M 1$ и $M 2$ пересекаются: $\operatorname{Re} k_{M 1}=\operatorname{Re} k_{M 2} \quad$ (а также $M 1^{\prime}$ и $\left.M 2^{\prime}: \operatorname{Im} k_{M 1} \approx \operatorname{Im} k_{M 2}\right)$. При этом параметр СЭВ $\tilde{\omega}_{L T}\left(\theta_{c}\right) / \Gamma=\omega_{L T} \sin ^{2} \theta_{c} / \varepsilon_{b} \Gamma_{c}$ для указанных критических углов принимает значения $0.1,0.183,0.399$. Это означает, что для критического угла выхода в вакуум $\theta_{c}=12^{\circ}$ $\left(\hbar \Gamma_{c} \approx 0.1 \mathrm{meV}\right)$ имеет место слабый, а для остальных двух значений $\theta_{c}-$ промежуточный поляритонный эффект. Поэтому на рис. $1, a^{\prime}$ в окрестности резонансной частоты $\omega_{c}$ практически не обнаруживается антипересечения дисперсионных кривых $M 1$ и $M 2$, а на рис. $1, b^{\prime}, c^{\prime}$ четко видна динамика образования нижных и верхних поляритонных ветвей на фоне сильной аномальной дисперсии.

Далее обсуждаем результаты численного расчета спектров люминесценции СЭПМ в окрестности критических значений затухания $\hbar \Gamma=\hbar \Gamma_{c}$ и угла выхода $\theta=\theta_{c}$ излучения в вакуум. При этом представляет наибольший интерес динамика развития спектральных кривых парциальных $I_{\beta}\left(\omega, \theta_{c}\right)$ и суммарной $I^{(0)}\left(\omega, \theta_{c}\right)$ интенсивностей, а также изменения механизмов формирования их в соответствии с дисперсией смешанных мод $M 1, M 1^{\prime}$ и $M 2, M 2^{\prime}$ в зависимости от значений $\hbar \Gamma_{c}, \theta_{c}$ и $\tilde{\omega}_{L T}$ в случаях $\Gamma \approx \Gamma_{c} \gg \tilde{\omega}_{L T}$ и $\Gamma \approx \Gamma_{c} \geq \tilde{\omega}_{L T}$. Парциальные вклады $I_{\beta}\left(\omega, \theta_{c}\right)$ на частоте $\omega=\omega_{c}$ не рассчитывались, так как они являются сингулярными величинами при $\Gamma=\Gamma_{c}$ на данной частоте. Однако при этом суммарная интенсивность $I^{(0)}\left(\omega, \theta_{c}\right)$ будет вполне измеряемая конечная величина, и можно рассчитать её с помощью формулы (6). Следует отметить, что интерференционная люминесценция СЭПМ при $\Gamma \approx \Gamma_{c}$ носит резонансный характер, т.е. в окрестности частоты $\omega_{c}$ с удалением от неё должно наблюдаться резкое уменьшение интерференционного эффекта, приводящего к узким спектральным линиям парциальных вкладов в общую интенсивность $I^{(0)}$.

Численный расчет парциальных спектров $I_{\beta}\left(\omega, \theta_{c}\right)$ проводился на компьютере с помощью программного обеспечения Matlab по формулам (16a) и (16b) работы [4]. При этом использовали те же значения экситонных параметров кристалла $\mathrm{CdS}$, как и на рис. $2, a^{\prime}, b^{\prime}, c^{\prime}$, и дополнили их значениями $l=70 \AA, L=0.80 \mu \mathrm{m}$.

На рис. 2, $a-c$ представлены рассчитанные спектральные зависимости общей, $I^{(0)}\left(\omega, \theta_{c}\right)$ (сплошные линии), и парциальных, $I_{\beta}\left(\omega, \theta_{c}\right)$, интенсивностей, обусловленных вкладами во внешнее излучение смешанных мод $\beta=M 1, M 2$ (пунктирные и штриховые кривые) и их интерференции $I_{M 12}(\beta=M 12$, штрихпунктирные линии) при тех же критических значенияях затухания $\hbar \Gamma=\hbar \Gamma_{c}$ и $\theta_{c}$, что и для дисперсионных кривых $a^{\prime}-c^{\prime}$.

Заметим, что, согласно рисункам, парциальные спектральные линии $I_{\beta}\left(\omega, \theta_{c}\right)$ при разных значениях $\Gamma_{c}$ качественно совпадают. Они имеют почти идентичные и резко асимметричные формы вблизи частоты $\omega_{c}$, полуширина которых слабо зависит от значения $\Gamma_{c}$. Суммарная кривая $I^{(0)}\left(\omega, \theta_{c}\right)$ в первом приближении, 
а $I_{12}\left(\omega, \theta_{c}\right)$ полностью симметричны относительно $\omega_{c}$. Это обусловлено тем, что параметр ПД для всех pис. $1, a-c$ имеет одинаковое значение $\gamma_{\text {пд }}=\Gamma_{c} / \Gamma \approx 10$. Однако при внимательном анализе указанных спектральных линий можно установить следующее. Во-первых, полуширина $\Delta_{A}$ спектральной линии $A_{L}$ результирующей интенсивности $I^{(0)}\left(\omega, \theta_{c}\right)$ сильно зависит от значений $\Gamma=\Gamma_{c}$ (или $\theta_{c}$ ) и $\tilde{\omega}_{L T}$, частота её максимума с ростом $\theta_{c}$ смещается в коротковолновую сторону от $\omega_{c}$ и, строго говоря, симметричный лоренцевский характер, справедливый при $\Gamma \approx \Gamma_{c} \gg \tilde{\omega}_{L T}$, нарушается. Во-вторых, особое внимание обращает на себя резко отличающие две части парциальных спектральных контуров $I_{M 1}\left(\omega, \theta_{c}\right)$ и $I_{M 2}\left(\omega, \theta_{c}\right)$ : очень сильные, узкие резонансные части полушириной $\Delta_{P} \approx 0.089 \mathrm{meV}$ над суммарным контуром $I^{(0)}\left(\omega, \theta_{c}\right)$ и относительно слабые, протяженные, резко асимметричные крылья. При этом резонансные, положительные парциальные вклады $I_{M 1}\left(\omega_{c}\right)+I_{M 2}\left(\omega_{c}\right)$ и отрицательный интерференционный вклад $I_{M 12}\left(\omega_{c}\right)$ сильно гасят друг друга и почти не влияют на полуширину $\Delta_{A}$ линии $A_{L}$. В-третьих, механизм формирования линии $A_{L}$ зависит от значения параметра СЭВ $\tilde{\omega}_{L T}\left(\theta_{c}\right) / \Gamma_{c}$. Так, уже при $\theta_{c}=12^{\circ}$ имеем $\tilde{\omega}_{L T} / \Gamma_{c} \approx 0.1$, и слабый поляритонный эффект приводит к сильной интерференции вблизи $\omega_{c}\left(-I_{12} \geq I_{M 1}, I_{M 2}\right)$ смешанных экситон-неоднородных волн, а при $\theta \geq 25^{\circ}$ преобладает роль СЭПМ $M 1$ и $M 2$. Суперпозиция этих двух мод с сильно асимметричными $I_{M 1}(\omega), I_{M 2}(\omega)$ и симметричными $I_{12}(\omega)$ контурами формирует практически симметричный спектральный контур $I^{0}\left(\omega, \theta_{c}=12^{\circ}\right)$ с максимумом на частоте $\omega_{c}$ (рис. $2, a$ ), и количественно хорошо воспроизводится экспериментальный спектр для $\mathrm{CdS}$ (треугольники). Расхождения теории и эксперимента на дальних краях спектра не превышают 5-7\%. Вчетвертых, с увеличением критического значения угла выхода $\left(\theta_{c}=24.5^{\circ}, 56^{\circ}\right)$ монотонно увеличивается параметр СЭВ $\tilde{\omega}_{L T} / \Gamma_{c} \approx 0.18,0.4$, и доминируют промежуточные смешанные поляритоны в формировании спектров люминесценции СЭПМ на фоне усиления аномальной дисперсии, что проявляется в существенном изменении максимальных значений интенсивностей $I_{\beta}\left(\omega, \theta_{c}\right)$ и слабой трансформации формы парциальных спектральных контуров (рис. $1, b, c)$.

На рис. 2 прослеживается четкая корреляция между дисперсионными кривыми $\left(a^{\prime}-c^{\prime}\right)$ и соответствующими спектральными линиями фотолюминесценции $(a-c)$ смешанных мод при разных критических значениях $\Gamma=\Gamma_{c}$. Видно, что динамика изменения дисперсии смешанных мод $M 1, M 2$ и $M 1^{\prime}, M 2^{\prime}$ в зависимости от значения $\Gamma_{c}$ вызывает адекватные изменения формы спектральных линий парциальных (кривые 1,2), интерференционных (кривые 12) и результирующих (кривые $\Sigma$ ) интенсивностей, обусловленные описанными выше особенностями.

\section{Заключение}

Таким образом, на основе полученных в настоящей работе результатов можно сделать следующий вывод. Рассчитанные ранее в работах $[3,4,7]$ спектры люминесценции при заданном значении затухания $\hbar Г$ позволяли следить за динамикой изменения спектров ЭПЛ с ростом $\theta$ при одновременном присутствии ПД $\left(\gamma_{\text {пд }}=\Gamma_{c}(\theta) / \Gamma\right)$ и СЭВ $\left(\gamma_{L T}=\tilde{\omega}_{L T}(\theta) / \Gamma\right)$. А представленный в настоящей работе совместный анализ дисперсионных кривых и спектров люминесценции СЭПМ демонстрирует одновременное влияние затухания и СЭВ на формирование спектров когерентного излучения сильно затухающих смешанных волн при постоянном значении параметра $\gamma_{\text {пд }}=1.0$. Этот анализ условий формирования спектральной линии $A_{L}$ позволяет утверждать, что процессы затухания экситонов влияют на её полуширину $\Delta_{A}$ сильнее, чем ПД.

\section{Благодарности}

Авторы выражают благодарность А.В. Селькину за предоставление экспериментальных результатов.

\section{Конфликт интересов}

Авторы заявляют, что у них нет конфликта интересов.

\section{Список литературы}

[1] Ахмедиев Н.Н. // ЖЭТФ. 1980. Т. 79. № 4 (10). С. 1534.

[2] Lebedev M.V., Lysenko V.G. // Sov. Phys. JETP. 1984. V. 59. N 6. P. 2193.

[3] Ивченко Е.Л., Селькин А.В., Абдукадыров А.Г., Сажин М.И., Юлдашев Н.Х. // Опт. и спектр. 1989. Т. 67. № 4. C. 845.

[4] Абдукадыров А.Г., Сажин М.И., Селькин А.В., Юлдашев Н.X. // ЖЭТФ. 1990. Т. 97. В. 2. С. 644.

[5] Masami Susaki, Kazuki Wakita, Nobuyuki Yamamoto. // Jpn. J. Appl. Phys. 1999. V. 38. N 5A. P. 1.

[6] Akhmadaliev B.Zh., Polvonov B.Z., Yuldashev N.Kh. // Opt. Spectrosc. 2014. V. 116. N 2. P. 244

[7] Akhmadaliev B.Zh., Yuldashev N.Kh., Yulchiev I.I. // Opt. Spectrosc. 2018. V. 125. N 3. P. 330.

[8] Юлдашев Н.X. Экситон-поляритонная люминесценция и перенос резонансного излучения в кристаллах. Фергана: Фаргона, 2002. 214 с. 